\title{
NATURAL AND ARTIFICIAL SCENTS DO NOT INCREASE EGG REJECTION RATES OF MODEL BROOD PARASITIC EGGS BY AMERICAN ROBINS (TURDUS MIGRATORIUS)
}

\author{
Mark E. Hauber \\ Department of Evolution, Ecology, and Behavior, School of Integrative Biology \\ University of Illinois at Urbana-Champaign, IL 61801, USA \\ E-mail: mhauber@illinois.edu,https://orcid.org/0000-0003-2014-4928
}

Hosts of obligate avian brood parasites can diminish or eliminate the costs of parasitism by rejecting foreign eggs from the nests. A vast literature demonstrates that visual and/ or tactile cues can be used to recognize and reject natural or model eggs from the nests of diverse host species. However, data on olfaction-based potential egg recognition cues are both sparse and equivocal: experimentally-applied, naturally-relevant (heterospecific, including parasitic) scents do not appear to increase egg rejection rates in two host species, whereas unnatural scents (human and tobacco scents) do so in one host species. Here I assessed the predictions that (i) human handling of mimetically-painted model eggs would increase rejection rates, and (ii) applying unnatural or natural scents to mimetically or non-mimetically painted model eggs alters these eggs' respective rejection rates relative to controls. I studied wild American Robins (Turdus migratorius), a robust rejecter species of the eggs of obligate brood parasitic Brown-headed Cowbirds (Molothrus ater). There was no statistical evidence to support either prediction, whereas poorer color-mimicry was still a predicted cause of greater egg rejection in this data set. Nonetheless, future studies could focus on this and other host species and using these and different methods to apply and maintain the scenting of model eggs longer to more directly test hosts' use of potential olfactory cues in the foreign-egg rejection process.

Key words: egg rejection, olfaction, parasitism, recognition systems, American Robins, Turdus migratorius

\section{INTRODUCTION}

In response to the often severe costs of brood parasitism (DAviEs 2000), many avian hosts reject foreign eggs from the nest (SEALY \& UNDERwOOD 2012). An extensive experimental literature has explored how the size (e.g., Rothstein 1982), weight (Ruiz-Raya et al. 2015), shape (Guigueno \& Sealy 2009), color (HAuber et al. 2019), maculation (Moskat et al. 2008), and/or surface texture (KEMAL \& Rothstein 1988) of natural or model eggs causes egg rejection within the same or across diverse host species. In turn, the putative sensory and cognitive systems of the hosts seem to have evolved to counter the anti-rejection adaptations by the parasites, namely their evolutionary shifts towards increasingly mimetic parasitic egg appearances (reviewed in Manna et al. 2017). 
Regarding the sensory modality of foreign egg recognition, the perception of visual (e.g., Stoddard \& Stevens 2010) and tactile (Tosi-German et al. 2020) cues has been identified in different host species. In contrast, experimental studies on the potential role of olfactory cues remain sparse in the host-parasite egg-rejection literature (MANNA et al. 2017). This is increasingly surprising since most birds, when tested, have fine-tuned olfactory perception (WhitTAKer et al. 2009) and are able to recognize many socio-ecologically relevant stimuli through volatile chemical cues (Wenzel 2007). A non-exclusive list of these recognition contexts includes: food (e.g., Nelson Slater \& HauBer 2017), conspecifics (Hagelin 2007), mates, (Bonadonna \& Nevitt 2004), own eggs (Goluke et al. 2016), and close kin (Coffin et al. 2011). Yet, there have been only a couple of studies, with contrasting results, that applied the experimental manipulation of odor(s) as the source(s) of egg recognition cues in host-parasite interactions (RASMUSSEN 2013, SOler et al. 2014). Regarding unnatural scents, human handling and strong tobacco scent both caused increased rejection of eggs in European Magpie Pica pica hosts of brood parasitic Great Spotted Cuckoos Clamator glandarius (Soler et al. 2014). However, the application of natural scents (this parasite's uropygial gland secretion or its cloacal lavage) did not alter egg rejection rates relative to controls (SOLER et al. 2014). Similarly, Song Thrush Turdus philomelos did not elevate the egg rejection rates of their own eggs when experimentally scented with heterospecific (New Zealand bellbird Anthornis melanura) uropygial oil (RAsmussen 2013).

Here I analyzed published data and conducted a brand new series of experimental trials on the egg rejection propensities of American Robins Turdus migratorius (hereafter: robins), a robust egg rejecter of obligate brood parasitic Brown-headed Cowbirds Molothrus ater (hereafter: cowbirds) (Luro et al. 2018). These analyses aimed to test both the role of unnatural (human handling; citrus scent) and natural (cloacal lavage of the brood parasite) scents. I chose a citrus (i.e., orange) scent as the unnatural odor application, because in at least one other avian species (i.e., Crested Auklets Aethia cristatella), a citrus (i.e., tangerine) scent was perceived and used for conspecific recognition (HAGELIN 2007). In turn, I painted model eggs with one of two different colours (known to elicit statistically different egg rejection rates by robins; deep blue: Luro and Hauber (2017) vs. mimetic robin-blue: Hauber et al. (2019) as positive controls, to determine whether the sample sizes applied in this study were sufficient to detect biological significance.

\section{METHODS}

In all of my laboratory's previous work on robins, we used naked (ungloved) human hands to handle the model eggs (once the experimental paint dried), until the egg was placed into the robin nest. Thus, our published data set(s) on mimetically painted model 
eggs can be used to assess whether human-handling increases egg rejection rates above the predicted nil $(0 \%)$ rate for mimetic, robin-blue model eggs. For this analysis, I selected a data set published by HAUBER et al. (2019) that was based on the egg rejection rate of one of that study's control treatment: that of cowbird egg-shaped, -sized, and -weighing, and mimetically-painted robin-blue, 3D-printed, model eggs.

For the scent-application experiments, I conducted a new set of trials. During MayJune, 2020, I located active nests of American Robins, with permission, throughout private orchards and tree farms near Urbana, IL, USA (for details of the study site and search methods, see HAUBER et al. 2020a). Nests were deemed active (i.e. during the laying and the incubation periods) when the clutch size increased on consecutive days, when a robin was flushed from the nest, or when the robin eggs were warm to human touch. In turn, robins are consistently robust egg rejecters of non-mimetic eggs throughout both the laying and incubation periods (АвоLINS-Aвоls \& HaUber 2020).

I used two different colors of 3D-printed, cowbird-sized, -shaped, and -weighing model eggs (sourced from www.Shapeways.com: "cow bird" egg in versatile natural plastic; for manufacture and dimension details, see IGIC et al. 2015): mimetic painted eggs were a robin-like blue color (paint-mix details sourced from CANNIFF et al. 2018), whereas nonmimetic, deep-blue eggs were painted with unmixed Ultramarine Winsor \& Newton Galeria Acrylic paint (London, UK; following Aвolins-Aвols \& HAUber 2020), both in triplicate coats. The resulting avian-perceived chromatic dissimilarity (just noticeable difference: JND, reviewed in STODDARD \& HAUber 2017) from natural robin egg colors was 2 JND for the mimetic eggs (HAUBER et al. 2019; 1-day egg rejection rate: 6.7\%) and 19 JND for the deep-blue eggs (Hauber et al. 2020b; rejection: 54\%) (Fig. 1).

For scent-application, I used a control (solvent only) and two treatments: the control was an even mix of unscented vegetable oil (Canola Oil, Meijers brand, USA) (50\%) and 95\% ethyl-alcohol (50\%). The unnatural scent treatment was an artificial citrus scent (Pure Orange Extract, McCormick brand, USA, dissolved in a mix of oil and aqueous ethyl-alcohol). Finally, the natural scent treatment was sourced from the separate cloacal lavages of 3 different adult female cowbirds captured during June 2020 (during the cowbirds' breeding season) at millet-baited potter traps near Urbana, IL, USA. I used $0.5 \mathrm{~mL}$ of the control solvent (see above) to inject and remove the solution from the cloaca of each of the 3 female cowbirds. These steps of lab-work were conducted while wearing gloves. The resulting liquid was then mixed with $0.5 \mathrm{~mL}$ of the solvent (see above) and stored at $4{ }^{\circ} \mathrm{C}$ overnights and then at ambient temperature during the 2-3 hrs of each morning's field work. To apply the control or treatment solutions upon the model eggs, I used a clean cotton tip dipped in the solution, covered the full surface of the model egg in the liquid, and then placed it in the robin's nest. This procedure delivered an average of $\sim 9 \mu \mathrm{g}$ solution atop each egg's surface.

Adult robins were not captured or marked in this study, and I used each nest as the unit of biological and statistical analysis. I conducted my experiments in bouts of 5-10 consecutive days, so that multiple, simultaneously active nests were experimented upon during the same bout, thus reducing potential pseudoreplication of studying the same breeding robin(s). All eggs were again handled by naked (ungloved) hands throughout the 2020 experiments.

Once a nest was located, it was exposed to 1-3 (median: 2) treatments/nest:

- control (solvent) applied to model mimetic egg $(n=10)$,

- control (solvent) applied to model non-mimetic egg $(\mathrm{n}=10)$,

- unnatural scent (citrus) applied to model mimetic egg $(n=14)$,

- unnatural scent (citrus) applied to model non-mimetic egg $(n=14)$,

- natural (cowbird cloacal lavage) scent applied to model mimetic egg $(\mathrm{n}=13)$. 

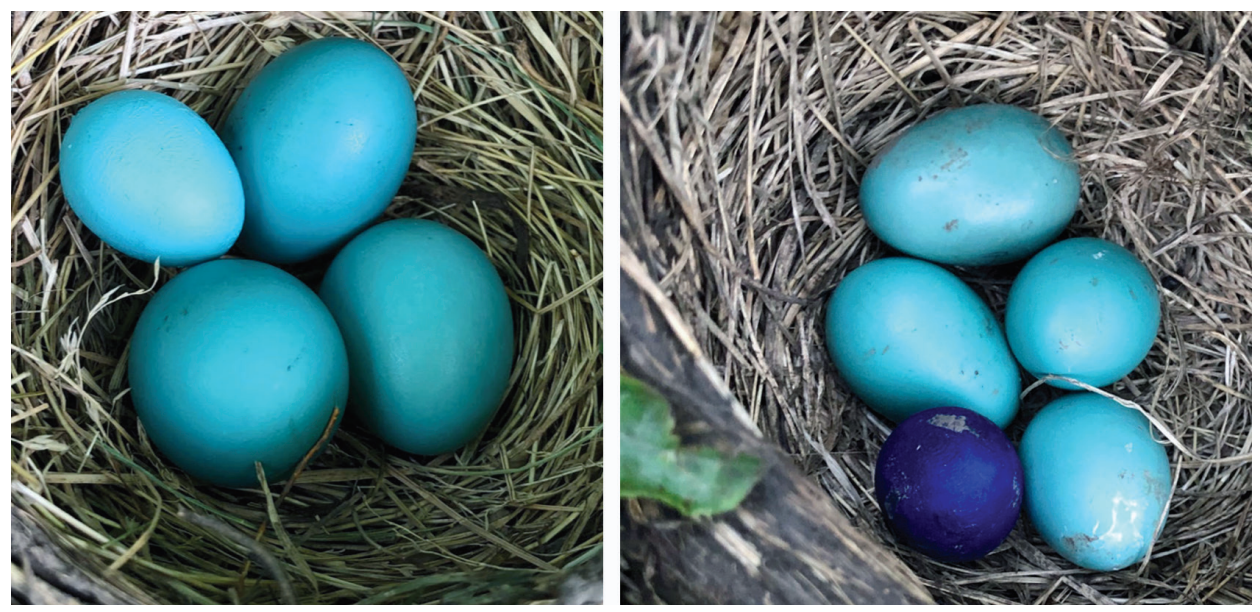

Fig. 1. Experimental clutches of American Robins, each with a robin-blue (mimetic; left) or a deep-blue (non-mimetic; right) model egg 3D printed in the size, shape, and weight of natural Brown-headed Cowbird eggs

I did not conduct a 6th treatment type of applying the natural cowbird scent to the non-mimetic model eggs, but this could be conducted informatively in a future study.

Robins are robust egg rejecters: in several of our previous studies we found that the median and mode for the latency of egg rejection of (e.g., non-mimetic: beige or deep-blue model eggs) is 1 day (Croston \& Hauber 2014a, Hauber et al. 2019). Therefore, I revisited each nest 1 day after the initial treatment and assessed whether the model egg was present (accepted) or missing (rejected) (as per Hauber et al. 2020b). I removed any model egg

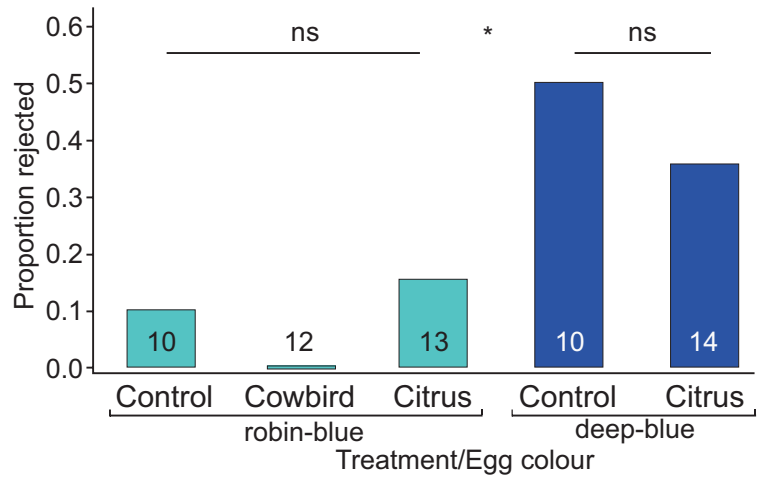

Fig. 2. Bivariate outcome of egg rejection trials 1 day after deploying the various model egg types. The * indicates a $p<0.05$ effect of model egg color; in turn, the olfactory treatments were not statistically significant predictors of egg rejection across or within colour treatments (ns). The numbers in the bars indicate the final sample sizes (\# of successful trials) still there, and then initiated the next, randomly chosen, different treatment at the same nest. Depredated or abandoned nests (cold eggs on two consecutive days) were removed from the analyses, as nest abandonment is not a response by American Robins to experimental brood parasitism (CROSTON \& HAUBER 2014b). The resulting sample sizes are indicated in Fig. 2.

For statistical analyses, I first used a one-sample onetailed sign test to determine whether human-handled robinmimetic eggs showed a rejection rate statistically greater than nil $(0 \%)$ given the sample size in a previously published experiment with these egg 
types. In turn, for the new experiments in this study, I used a two-tailed nominal logistic mixed model in JMP 12.0 (SAS Institute, Cary, USA) on the bivariate outcome of each trial (accepted, rejected) with experiment order (per nest), treatment type (natural scent, unnatural scent, or control), painted color (mimetic or non-mimetic) and the interaction term of these latter two included as predictor variables, nest ID used as a random effect, and $\alpha=$ 0.05 . The results were statistically equivalent when reanalyzing the data in the $\mathrm{R}$ Statistical Package (R Core Team 2017).

\section{RESULTS}

I detected no statistical difference from a predicted nil $(0 \%)$ rejection of human-handled, mimetically painted model eggs in a previously published data set (rejection rate: $6.7 \%, \mathrm{n}=15 ; \mathrm{p}=0.16$ ).

Similarly, experimental scent treatment did not statistically alter the egg rejection rates of model eggs relative to controls $\left(\chi^{2}=2.6, p=0.27\right)$; in contrast, as predicted, non-mimetic deep-blue model eggs were rejected significantly more often than mimetic robin-blue eggs $\left(\chi^{2}=5.5, \mathrm{p}=0.019\right)$, irrespective of experiment order $\left(\chi^{2}=1.2, \mathrm{p}=0.28\right)$ and the interaction term of treatment and color $\left(\chi^{2}=0.59, \mathrm{p}=0.44\right)$ (Fig. 2).

\section{DISCUSSION}

The experimental treatments applied in this study did not yield statistical support for the role of olfactory cueing for egg rejection responses by American Robins to model eggs. In the mimetic-egg color treatment, with the models handled directly, rejection rates were not statistically higher than nil. In turn, the application of neither unnatural nor natural scents caused greater model egg rejection rates relative to the control (solvent) treatments. However, the sample sizes of these experiments were sufficient to detect the statistical effect of greater rejection rates of non-mimetic relative to mimetic model egg colors (Fig. 2).

Methodologically, a concern of the experimental manipulations applied here may be that the volatile component of the control and treatment scenting did not last long enough to be perceived sufficiently and cause egg rejection by incubating robins. Video-recordings show that robins returning to experimental nests often touch the new, model eggs with their beak, but they can still take typically more than 1-2 hrs to reject even non-mimetic, including cowbird-like, model eggs (Hauber et al. 2019, Scharf et al. 2019); therefore, any experimental olfactory cue must, in principle, linger in a sufficient concentration for longer than that, too. Perhaps a 3D-printed model egg with a porous surface and an internal scent-solvent reservoir might need to be designed to achieve this effect over a longer timescale. 
In turn, it is possible that the choice of host species for the handful of olfactory egg recognition studies conducted to-date may have been constrained in that all three study species were open-cup nesting hosts, with sufficient environmental light-exposure in their nests to allow visually-based egg foreign recognition. Furthermore, the focal species in the present study, the American Robin, was also not odor-sensitive in a previous set of experiments assessing the role of sensory cues for foraging strategies (HepPNER et al. 1965). Instead, therefore, future studies should focus on manipulating the odor profiles of hosts of brood parasitic birds that reproduce in dark milieus, including those in enclosed nests and cavities (e.g. Tosi-German et al. 2020), where olfactory cues might become more salient for egg discrimination. Accordingly, RasmusSEN (2013) showed that the uropygial oil's chemical profile of the enclosednest breeding Grey Warbler Gerygone igata was matched by that of its local specialist brood parasite, the Shining Bronze Cuckoo Chalcites basalis, in New Zealand. Alternatively, or in addition, olfactory-cued egg recognition may also be more prominent in host species where parasitic eggshell size, shape, color, and maculation mimicry has reached such high levels at which avianperceivable visual cues are no longer discriminable between own vs. foreign eggs (e.g. Red-backed Shrikes Lanius collurio: Lovaszi \& Mosкат 2004, Chaffinches Fringilla coelebs: Sтокке et al. 2004, Common Redstarts Phoenicurus phoenicurus: Manna et al. 2020).

Overall, these results parallel previous findings that the application of naturally-relevant (parasitic or other heterospecific scent) does not increase the rejection rates of eggs in nests of otherwise egg-rejecter hosts of avian brood parasites (RASMUSSEN 2013, Soler et al. 2014). In turn, the outcomes of applying unnatural scents (human handling and citrus) here did not match a previous report's outcomes from such treatments (Soler et al. 2014). Methodological improvements for the delivery of scent cues, direct behavioral observations of hosts' responses at the nests, and the choice of additional (e.g., cavity-nesting) host species are still required for future work to more fully assess the potential role of olfactory cues in avian host-parasite arms-races.

Acknowledgements - For access to robin nests, I thank S. Winnicki, O. Rhodes, J. Hoover, and the owners of Wandell's and Country Arbors Tree Farms and Nurseries in Urbana, IL, USA. Research for this study was approved by institutional, state, and federal agencies. I thank E. Ngumbi for the use of lab equipment, J. Rasmussen and J. Briskie for access to the unpublished PhD thesis at the University of Canterbury, New Zealand, and D. Whittaker, D. Hanley, K. Summers, J. Hoover, F. Hoover, W. Schelsky, H. Pollock, and others for helpful discussions. Funding was provided by the Harley Jones Van Cleave Professorship at the University of Illinois. This research was permitted by institutional, state, and federal agencies. I am also grateful to the editor and the referees for constructive comments. 


\section{REFERENCES}

Aвolins-Abols, M. \& Hauber, М. E. (2020): Proximate predictors of variation in egg rejection behavior by hosts of avian brood parasites. - Journal of Comparative Psychology Advance online publication. https://doi.org/10.1037/com0000225

Bonadonna, F. \& Nevitt, G. A. (2004): Partner-specific odor recognition in an Antarctic seabird. - Science 306: 835. https://doi.org/10.1126/science.1103001

Canniff, L., Dainson, M., Lopez, A. V., Hauber, M. E., Grim, T., Samas, P. \& Hanley, D. (2018): Probing the limits of egg recognition using egg rejection experiments along phenotypic gradients. - Journal of Visualized Experiments 138: e57512. https://doi.org/10.3791/57512

Coffin, H. R., Watters, J. V. \& Mateo, J. M. (2011): Odor-based recognition of familiar and related conspecifics: A first test conducted on captive Humboldt Penguins (Spheniscus humboldti). - PLoS ONE 6: e25002. https://doi.org/10.1371/journal.pone.0025002

Croston, R. \& Hauber, M. E. (2014a): High repeatability of egg rejection in response to experimental brood parasitism in the American robin (Turdus migratorius). - Behaviour 151: 703-718. https://doi.org/10.1163/1568539X-00003164

Croston, R. \& Hauber, M. E. (2014b): Spectral tuning and perceptual differences do not explain the rejection of brood parasitic eggs by American robins (Turdus migratorius). - Behavioral Ecology and Sociobiology 68: 351-362. https://doi.org/10.1007/s00265-013-1649-8

Davies, N. B. (2000): Cuckoos, cowbirds, and others cheats. - T \& A D Poyser, London, 328 pp.

Goluke, S., Dorrenberg, S., Krause, E. T. \& Caspers, B. A. (2015): Female zebra finches smell their eggs. - PLoS ONE 11: e0155513. https://doi.org/10.1371/journal.pone.0155513

Guigueno, M. F. \& Sealy, S. G. (2009): Nest sanitation plays a role in egg burial by yellow warblers. - Ethology 115: 247-256. https://doi.org/10.1111/j.1439-0310.2008.01597.x

Hagelin, J. C. (2007): The citrus-like scent of crested auklets: reviewing the evidence for an avian olfactory ornament. - Journal of Ornithology 148: S195-S201. https://doi. org/10.1007/s10336-007-0185-6

Hauber, M. E., Dainson, M., Luro, A., Louder, A. A. \& Hanley, D. (2019): When are egg rejection cues perceived? A test using thermochromic eggs in an avian brood parasite host. - Animal Cognition 22:1141-1148. https://doi.org/10.1007/s10071-019-01306-w

Hauber, M. E., Abolins-Abols, M., Kiм, C. R. \& Paitz, R. T. (2020a): Inter-individual variation in anti-parasitic egg rejection behavior: a test of the maternal investment hypothesis. - Integrative Organismal Biology 2: obaa014. https://doi.org/10.1093/iob/obaa014

Hauber, M. E., Kim, C. R., Goethe, C. \& Hanley, D. (2020b): Self-referent phenotype matching is a poor predictor of egg rejection by American Robins. - Journal of Field Ornithology 91: 254-262. https://doi.org/10.1111/jofo.12339

HeppNer, F. (1965): Sensory mechanisms and environmental clues used by the American Robin in locating earthworms. - Condor 67: 247-256. https://doi.org/10.2307/1365403

Igic, B., Nunez, V., Voss, H. U., Croston, R., Aidala, Z., Lopez, A.V., van Tatenhove, A., Holford, M. E., Shawkey, M. D. \& Hauber, M. E. (2015): Using 3D printed eggs to examine the egg-rejection behaviour of wild birds. - PeerJ 3: e965. https://doi. org/10.7717/peerj.965

Kemal, R. E. \& Rothstein, S. I. (1988): Mechanisms of egg recognition: adaptive responses to eggs with broken shells. - Animal Behaviour 36: 175-183. https://doi.org/10.1016/ S0003-3472(88)80261-6

Lovaszi, P. \& Moskat, C. (2004): Break-down of arms race between the red-backed shrike (Lanius collurio) and common cuckoo (Cuculus canorus). - Behavior 141: 245-262. https://doi.org/10.1163/156853904322890843 
Luro, A. B. \& Hauber, M. E. (2017): A test of the nest sanitation hypothesis for the evolution of foreign egg rejection in an avian brood parasite rejecter host species. - The Science of Nature 104: 14. https://doi.org/10.1007/s00114-017-1446-8

Luro, A., Igic, B., Croston, R., Lopez, A. V., Shawkey, M. D. \& Hauber, M. E. (2018): Which egg features predict egg rejection responses in American robins? Replicating Rothstein's (1982) study. - Ecology E Evolution 8: 1673-1679. https://doi.org/10.1002/ece3.3759

Manna, T. J., Hanley, D., Honza, M., Capek, M., Rutila, J., Samas, P., Abolins-Abols, M. \& Hauber, M. E. (2020): Fitting different visual models to behavioral patterns of parasitic egg rejection along a natural egg color gradient in a cavity-nesting host species. - Vision Research 167: 54-59. https://doi.org/10.1016/j.visres.2019.12.007

Manna, T., Moskat, C. \& Hauber, M. E. (2017): Chapter 24: Cognitive decision rules for egg rejection. Pp. 438-448. In: Soler, M. (ed.): Avian brood parasitism. - Springer Nature, New York. https://doi.org/10.1007/978-3-319-73138-4_24

Moskát, C., Székely, T., Cuthill, I. C. \& Kisbenedek, T. (2008): Hosts' responses to parasitic eggs; which cues elicit hosts' egg discrimination? - Ethology 114: 186-194. https://doi. org/10.1111/j.1439-0310.2007.01456.x

Nelson Slater, M. \& Hauber, M. E. (2017): Olfactory enrichment and scent cue associative learning in captive birds of prey. - Zoo Biology 36: 120-126. https://doi.org/10.1002/zoo.21353

R Core Team (2017): R: A language and environment for statistical computing. - R Foundation for Statistical Computing, Vienna. https://www.R-project.org/

RAsmussen, J. L. (2013): Investigations of evolutionary arms races and host diversity in avian brood parasite systems. - Unpublished PhD Dissertation, University of Canterbury, Christchurch, New Zealand.

Rothstein, S. I. (1982): Mechanisms of avian egg recognition: which egg parameters elicit responses by rejecter species? - Behavioral Ecology and Sociobiology 11: 229-239. https:// doi.org/10.1007/BF00299299

Ruiz-Raya, F., Soler, M., Sanchez-Perez, L. L. I. \& Ibanez-Alamo, J. D. (2015): Could a factor that does not affect egg recognition influence the decision of rejection? - PLoS ONE 10: e0135624. https://doi.org/10.1371/journal.pone.0135624

Scharf, H. M., Stenstrom, K., Dainson, M., Benson, T. J., Fernandez-Juricic, E. \& HauBER, M. E. (2019): Mimicry-dependent lateralization in the visual inspection of foreign eggs by American robins. - Biology Letters 15: 20190351. https://doi.org/10.1098/ rsbl.2019.0351

SEALY, S. G. \& Underwood, T. J. (2012): Egg discrimination by hosts and obligate brood parasites: a historical perspective and new synthesis. - Chinese Birds 3: 274-294. https:// doi.org/10.5122/cbirds.2012.0042

Soler, J. J., Perez-Contreras, T., De Neve, L., Macias-Sanchez, E., Moller, A. P. \& Soler, M. (2014): Recognizing odd smells and ejection of brood parasitic eggs. An experimental test in magpies of a novel defensive trait against brood parasitism. - Journal of Evolutionary Biology 27: 1265-1270. https://doi.org/10.1111/jeb.12377

Stoddard, M. C. \& Stevens, M. (2011): Avian vision and the evolution of egg color mimicry in the common cuckoo. - Evolution 65: 2004-2013. https://doi.org/10.1111/j.15585646.2011.01262.x

Stoddard, M. C. \& Hauber, M. E. (2017): Colour, vision and coevolution in avian brood parasitism. - Philosophical Transaction of the Royal Society of London B 372: 20160339. https://doi.org/10.1098/rstb.2016.0339

Stokke, B. G., Rudolfsen, G., Moksnes, A. \& Roskaft, E. (2004): Rejection of conspecific eggs in chaffinches: The effect of age and clutch characteristics. - Ethology 110: 459470. https://doi.org/10.1111/j.1439-0310.2004.00978.x 
Tosi-German, A., Tassino, B. \& Reboreda, J. C. (2020): Female and male rufous horneros eject shiny cowbird eggs using a mental template of the size of their own eggs. - Behavioural Processes 178: 104152. https://doi.org/10.1016/j.beproc.2020.104152

Wenzel, B. M. (2007): Avian olfaction: then and now. - Journal of Ornithology 148: S191S194. https://doi.org/10.1007/s10336-007-0147-z

Whittaker, D. J., Reichard, D. G., Dapper, A. L. \& Ketterson, E. D. (2009): Behavioral responses of nesting female dark-eyed juncos Junco hyemalis to hetero- and conspecific passerine preen oils. - Journal of Avian Biology 40: 579-583. https://doi.org/10.1111/ j.1600-048X.2009.04813.x

Received August 30, 2020, accepted October 7, 2020, published November 13, 2020 
